In: Textbook of Schizophrenia Spectrum and Related Disorders, edited by M. Ritsner, in press, August 2010

\title{
One hundred years of insanity: genomic, psychological, and evolutionary models of autism in relation to schizophrenia
}

\section{Bernard J. Crespi}

Department of Biosciences, Simon Fraser University, Canada

\begin{abstract}
The Swiss psychiatrist Eugen Bleuler coined the terms 'schizophrenia', for the splitting of psychic functions, and 'autism', for withdrawal from external reality in patients with schizophrenia, almost exactly a century ago. Ever since 1943 when Leo Kanner co-opted 'autism' to refer to a new condition involving 'disturbance of affective contact' manifested in children, the relationship between schizophrenia and Kanner's autism has remained unclear. In this article I draw on data from studies of genomics, neurodevelopment, psychology, psychiatry and evolutionary biology to develop and evaluate alternative hypotheses for the relationship between autism and schizophrenia spectrum conditions. These data provide evidence for two hypotheses: partially-overlapping etiology of autism with schizophrenia mediated by common risk factors, and diametric causes of autism and schizophrenia mediated by genes underlying under-development versus dysregulated over-development of human social-brain phenotypes. The primary
\end{abstract}


practical implications of these results are that: (1) false-positive diagnoses of premorbidity to schizophrenia-spectrum conditions as autistic spectrum conditions may be common, and may indicate a structural flaw in current diagnostic, nosological frameworks, (2) schizophrenia may be due in part to losses of function in negative regulators of social cognition and affect, rather than 'deficits' in brain development; (3) the development of new pharmacological treatments for autism and schizophrenia can benefit directly from models of how autistic spectrum and schizophrenia spectrum conditions are etiologically related; and (4) future studies of autism, schizophrenia, and their relationship to one another must increasingly seek to integrate across analytic levels, from genes to neurodevelopment, neurological function, neuroanatomy, cognition, and evolutionary biology of the social brain, in the context of subtyping the substantial genetic and clinical heterogeneity found in each set of conditions.

\title{
Key words
}

Schizophrenia, autism, genomics, nosology, evolution, social brain

\author{
Abbreviations \\ DSM - Diagnostic and Statistical Manual of Mental Disorders \\ PDD-NOS - Pervasive Developmental Disorder - Not Otherwise Specified
}




\section{Introduction}

The great Colombian author Gabriel García Márquez reified the cyclical nature of history in his Nobel Prize-winning 1967 book, One Hundred Years of Solitude. Eugen Bleuler's less-famous book Dementia Præcox or the Group of Schizophrenias ${ }^{1}$, originally published in 1911 , saw first use of the term 'autism', a form of solitude manifest by Bleuler as withdrawal from reality in schizophrenia. This neologism, about to celebrate its centenary, epitomizes an astonishing cycle of reification and change in nosology, a cycle only now coming into clear view as molecular-genetic data confronts the traditional, age-old categories of psychiatric classification.

The purpose of this article is to review and evaluate the relationship of schizophrenia with autism, to help illuminate the causes of both conditions. To do so, I integrate data from recent studies of genomics, neurodevelopment, psychology, and psychiatry, in the context of alternative hypotheses for how schizophrenia and autism are related to one another, genetically, developmentally, psychologically, and with regard to the evolution of socialcognitive and social-affective phenotypes in humans.

I first provide a brief history of the term 'autism', in the context of schizophrenia, Kanner's autism, and diagnostic methods. This background is necessary for understanding how 'autism' and 'autistic' are currently conceived and applied in the literature.

Second, I review evidence regarding the phenotypes and phenotypic structure of autism, and schizophrenia, and how they relate to diagnostic 
classifications. I introduce the concept of 'positive' symptoms as manifestations of autism, to help in contrasting autism with schizophrenia psychiatrically, psychologically, and with regard to underlying neurodevelopment, physiology, and brain morphology and function.

Third, I describe alternative models for how schizophrenia-spectrum conditions and autism are related to one another psychologically, developmentally, neurologically, and with regard to evolution of the human social brain. Each of these models makes specific predictions that can be tested in a strong-inference framework, using data on causes, correlates and consequences of variation in neurodevelopment.

Finally, I discuss the primary implications of this review for practical applications, including pharmacological therapy, strategies for uncovering risk genetic risk factors, integration of etiological studies across causal levels from genes to psychiatric phenotypes, and conceptualization of psychiatric conditions in relation to normative development.

\section{'Autism' from Bleuler to the DSM}

One of the most important symptoms of schizophrenia is the preponderance of inner life with an active turning-away from the external world. The most severe cases withdraw completely and live in a dream world; the milder cases withdraw to a lesser degree. I call this symptom "autism." (p. 397) 
The outstanding, "pathognomonic", fundamental disorder is the children's inability to relate themselves in the ordinary way to people and situations from the beginning of life. ... This is not, as in schizophrenic children or adults, a departure from an initially present relationship; it is not a "withdrawal" from formerly existing participation. From the start there is an extreme autistic aloneness. ${ }^{2}$

Kanner ${ }^{2}$ struggled to establish 'autism' as a condition separate from schizophrenia, especially given the tendency among psychiatrists to consider schizophrenia as some function of Bleuler's 'four As' of autism, association, affect, and ambivalence ${ }^{3}$. Kanner's attempts to conceptually-extract autism from schizophrenia initially failed, and throughout much of the 1950s, 1960s and 1970s, autism was considered as a manifestation or subtype of schizophrenia in children ('childhood-onset' schizophrenia) ${ }^{4,5}$. Eventually, documentation by Kolvin ${ }^{6}$ of a bimodal onset or diagnosis of autism (in early childhood) and schizophrenia (in adolescence), and comprehensive reviews by Rutter ${ }^{7-9}$ contrasting the correlates and phenotypes of autism and schizophrenia, validated autism as a separate and distinct condition. This condition was formalized by DSM-III in the context of 'infantile autism' as a subgroup of 'pervasive developmental disorder' ${ }^{\prime 10}$. The initial diagnostic criteria for 'infantile autism' included: (1) lack of responsiveness to others, (2) language absence or abnormalities, (3) resistance to change or attachment to objects, (4) the absence of schizophrenic features, and (5) onset before 30 months. A more-flexible DSMIII-R, based in large part on work by Rutter' ${ }^{9}$, saw the first formalization, in 'autistic 
disorder', of the familiar triad of impairments: (1) reciprocal social interaction; (2) verbal and non-verbal communication; (3) restricted repertoire of activities and interests; with diagnostic thresholds based on minimum numbers of specific behaviors in each category, and overall.

DSM-IV was fundamentally similar to DSM-III, but with the addition, among other diagnoses, of PDD-NOS (pervasive developmental disorder not otherwise specified). PDD-NOS is defined as 'severe and pervasive impairment in the development of reciprocal social interaction or verbal and nonverbal communication skills, or when stereotyped behavior, interests, and activities are present", when the full criteria are not met for autistic disorder or other conditions (including schizophrenia and schizotypal personality disorder), due to late age of 'onset' (after age 3), or atypical or sub-threshold symptoms.

Finally, nascent DSM-V simplifies diagnostics by subsuming the full set of closely-related idiopathic autism spectrum conditions (autistic disorder, Asperger's syndrome, and PDD-NOS) under a single category, 'autistic disorder', with three criteria: (1) persistent deficits in social communication, reciprocity, and peer interactions, (2) restricted, repetitive patterns of behavior, interests and activities, and (3) symptoms present in early childhood but possibly 'not fully manifest until social demands exceed limited capacities'.

Most modern research on autism spectrum conditions, and their relationship to schizophrenia, has been conducted under the rubrics of DSM-IV, which was published in 1994. Under these criteria, PDD-NOS, considered as an autism spectrum condition, applies to any child with social-behavorial 
impairments salient but sub-threshold to autistic disorder. The PDD-NOS category has been applied very extensively, with diagnostic prevalence comparable of that of autism and Asperger's syndrome combined in some populations ${ }^{11}$, probably due in part to its lack of specificity ${ }^{12}$. About half of PDDNOS cases involve clinical social and communication deficits, but sub-threshold impairments in repetitive behavior and restricted interests ${ }^{13}$.

With regard to diagnostic accuracy, and conceptualization of how autism is related to schizophrenia from a neurodevelopmental perspective, two key questions follow from work conducted under DSM-IV: how often premorbidity to schizophrenia is sufficiently severe to result in autism spectrum diagnoses during childhood, and how often schizophrenia that lacks prominent positive symptoms has been diagnosed as autism spectrum or considered 'autistic'. These questions will be addressed below, in the contexts of information on manifestations of premorbidity to schizophrenia, and genetic data on the causes of schizophrenia and autism.

Despite the clear separation of schizophrenia from autism provided by all versions of the DSM, Bleuler's concept of 'autistic' deficits in schizophrenia has persisted in the literature, probably due to the prominent impairments in social reciprocity, language, and theory of mind found in most schizophrenic individuals ${ }^{14-18}$. Such impairments are, moreover, frequently combined with elements of obsessive or compulsive behaviors ${ }^{19}$, as is autism ${ }^{20}$. These phenotypes of schizophrenia correspond closely, of course, with the tried of traits used to describe and diagnose autism. Conflation of schizophrenia with autism 
can usually be avoided by the frequent presence of prominent 'positive' symptoms, such as hallucinations, delusions, and thought disorder, characteristic of schizophrenia and exclusionary of autism in the DSM. However, such positive symptoms are also highly variable and episodic, and patients with schizophrenia or schizotypy typified by predominantly negative and disorganized symptoms overlap in their profiles of psychological and behavioral symptoms with some autistics $^{21-25}$; such individuals are also, like autistics, most-commonly male ${ }^{26}$.

These considerations suggest that many schizophrenics and individuals with schizotypal personality disorder are likely to score in the 'autism' or 'autism spectrum' (including PDD-NOS) ranges on DSM-based or other metrics of 'autistic' symptoms, as demonstrated by several studies ${ }^{22,27,28}$. Of such metrics, the most inclusive is represented by the 'broad autism phenotype'29, a set of language and personality traits that are similar to, but milder than, those found in autism. Comparison of the broad autism phenotype questionnaire ${ }^{29}$ to its equivalent in the study of schizophrenia, the schizotypal personality questionnaire $^{30-32}$, indicates considerable similarity between measures of the broad autism phenotype and the interpersonal and disorganized dimensions of schizotypy. Such resemblances are validated by scores above the broad autism phenotype cutoff for schizophrenic and schizotypal individuals in one study ${ }^{33}$, and supported by similarities between questionnaire measures of Asperger's syndrome and schizotypy ${ }^{23}$. These similarities and possible 'false-positive' diagnoses apparently reflect the central roles of deficits in social cognition and 
behavior, and unusual patterns of interests and compulsions, among both the autism and schizophrenia spectra, regardless of their underlying causes.

Deficits in sociality and language, and the presence of repetitive behavior and restricted interests, usually remain idiopathic, but are also not uncommon in a variety of neurogenetic conditions, such as Velocardiofacial syndrome, Klinefelter syndrome and Prader-Willi syndrome, that involve neurological phenotypes of schizophrenia in a large subset of individuals, and a high incidence of schizophrenia and schizotypy in adults ${ }^{34-45}$. Individuals with such syndromes - which are usually recognized in childhood - have often been diagnosed as 'autistic' if they meet all three autism criteria, or 'autism spectrum' (usually PDD-NOS) if the fit between phenotypes and criteria is less complete. 'Autism spectrum' diagnoses are thus usually applied, in both idiopathic and syndromic cases, to children who are under the usual age (in the late teens) at which schizophrenia is expressed. As a result, individuals who are 'premorbid' for schizophrenia are not diagnosed as such (due to a lack of specific, predictive criteria for later development of schizophrenia), but are categorized as 'autism spectrum' if their social, language, and/or repetitive-behavioral restricted-interest impairments are sufficiently severe. Social, language, and other developmental deficits and delays are indeed common among children - especially boys - who later develop schizophrenia ${ }^{27,46-54}$. How often do such phenotypes motivate childhood diagnoses of 'autism spectrum'?

The frequency of 'false-positive' autism spectrum diagnoses ${ }^{55}$ among preschizophrenic or schizotypal children remains unclear, especially for individuals 
without identified genetic or genomic risk factors. However, the tendencies for genomic copy number variants to exert severe effects on diverse aspects of early neurodevelopment ${ }^{56}$, and for schizophrenia of earlier onset to exhibit a higher male sex-ratio bias and a stronger tendency to be associated with copy-number variants rather than other factors ${ }^{5,57}$ suggests a substantial risk for false-positive diagnoses of autistic spectrum conditions $s^{4,28,33,49,55}$ at least for individuals with genomic or genetic risk factors of high penetrance. The most direct evidence for diagnostic conflation of the autism spectrum with premorbidity to schizophrenia, schizotypy, or other schizophrenia-spectrum conditions comes from diagnoses of autism spectrum conditions in children with deletions at $15 q 11.2,15 q 13.3$, and 22q11.21, and duplications of 16p11.2, copy number variants for which schizophrenia risk has been well-established from studies of adults ${ }^{36,49,58-62}$. By contrast, autism-associated copy number variants, such as deletions at $16 \mathrm{p} 11.2$ ${ }^{63}$, or duplications at $22 q 11.21^{64}$ have seldom also been reported in individuals diagnosed with schizophrenia and have not been established as risk factors.

Differentiating autism spectrum conditions from false-positive diagnoses of premorbidity to schizophrenia requires judicious use of intermediate, biologicallybased phenotypes including 'positive' symptoms of autism (as described below), adoption of relatively-new diagnostic categories such as Multiple Complex Developmental Disorder ${ }^{65,66}$, and use of data from genomic risk factors in developmental, longitudinal perspectives. In particular, children with diagnoses of autism spectrum conditions who harbour deletions at 15q11.2, 15q13.3, or 22q11.21, duplications at 16p11.2, supernumary X chromosomes (Klinefelter 
syndrome in males), paternal deletions of $15 q 11-13$ or maternal uniparental disomy for chromosome 15 (Prader-Willi syndrome), or other well-validated schizophrenia-spectrum genomic risk factors, might usefully be monitored for the development of schizophrenia spectrum conditions after the onset of adolescence, and studied for the presence of biologically-based schizophreniaspectrum phenotypes ${ }^{43,44,62,67}$. To the degree that false-positive diagnoses of autism spectrum conditions in childhood are not uncommon, and autism and schizophrenia are underlain by different genetically-based risk factors, inclusion of children premorbid for schizophrenia in studies to uncover novel genetic or genomic risk factors for autism will dilute the probability of detecting significant results.

Consideration of autism from the general perspective of impaired social interaction, due to some combination of deficits in reciprocity and language, and repetitive, restrictive interests and behavior, cycles us back to Blueler's original incarnation. The extensive cognitive-affective and behavioral variation among children with Kanner's autism has motivated, justifiably, a wide diagnostic net but such a net, especially when structured mainly by the presence of deficits, may capture individuals with impairments due to highly diverse causes.

Ultimately, the diagnostic bases of autism and schizophrenia should presumably be founded on models of causation, models that can subsume the diversity of developmental, physiological, morphological, behavioral and psychological phenotypes found in each condition under a relatively small set of convergent frameworks. Only in this way can the diagnostic categorizations of autism and 
schizophrenia each be reconciled with their genetic and neurodevelopmental foundations, and nosology be connected with proximate and ultimate causes ${ }^{68}$. These are fundamentally important issues because, as described below, many researchers consider autism and schizophrenia to be similar conditions based on the centrality of social deficits in both, while others consider them to be separate and independent (as in the DSM), or diametric.

\section{Phenotypic structure of autism and schizophrenia spectrum}

Reification of autism and schizophrenia as 'real' categories, rather than pragmatic human constructs, may be a natural consequence of their fundamental, long-term historical role in structuring one hundred years of thought and research into causes of human psychiatric conditions. The usefulness to society of these constructs has been extensively demonstrated, but evaluation of their status as dimensional versus categorical, and causally divisible into components versus unitary, has required data on the genetic bases of the conditions, their sets of diagnostic criteria, and the relationships between their component phenotypes. Such information has become available and begun to solidify mainly within the past few years, allowing the first explicit comparisons between the phenotypic structures of schizophrenia and autism.

First, schizophrenia (and related conditions) and autism each encompass sets of dissociable but more or less related phenotypes. Schizophrenia is not clearly separable from bipolar disorder and major depression; these conditions overlap broadly in core phenotypes, exhibit joint familial aggregation even when 
underlain by single, segregating penetrant risk factors such as DISC1 loss of function, and are mediated by genetic risk factors that may influence liability to pairs or trios of the conditions, or specific symptoms such as aspects of psychosis $^{69-72}$. Considered together, these conditions are thus referred to as the 'schizophrenia spectrum', or 'psychotic-affective spectrum', and considered as a set of genetically and phenotypically related conditions comprising a mosaic of partially-overlapping but dissociable psychotic, manic, affective and other symptoms. Any given individual can be conceptualized as occupying a zone in three-dimensional space defined by degree of expression of psychotic (including positive, negative and disorganized), manic and affective phenotypes, or, more specifically by a multidimensional space that comprises the constellation of phenotypes found in schizophrenia, bipolar disorder, and major depression. Van Os ${ }^{73}$ provides a useful depiction of such a space, in the context of 'salience syndrome' as a unified framework for understanding phenotypes associated with psychosis. Phenotypes of the schizophrenia spectrum are dissociable, in that specific symptoms can and do occur in isolation, and components are relatively independent genetically ${ }^{74,75}$ - but they often occur together, presumably due to pleiotropy and shared neurodevelopmental causes. However, the relationship of psychotic, manic, affective, and other schizophrenia-spectrum symptoms to one another remains poorly understood.

The trio of phenotypes diagnostic of idiopathic autism has been demonstrated to be partially if not largely dissociable, in that the phenotypic and genetic correlations between measures of impairments in social reciprocity, 
language, and restrictive, repetitive behavior and interests are modest ${ }^{12,76,77}$. Autism can thus be considered as a set of social and behavioral traits that are genetically and phenotypically related in that they occur together somewhat more often than expected by chance, or interact additively or in synergy to produce a recognizable pattern, but are also separable, in that any specific component of the three can and does occur in isolation. Any given individual can thus be conceptualized as occupying some area in a multi-dimensional space with axes defined by the diagnostic triad and their severities ${ }^{12}$. The relationship of social with non-social phenotypes remains poorly understood ${ }^{78}$, and the degree to which the three core components of autism would emerge from factor analysis of a much wider range of childhood behavioral phenotypes than previously included $^{77}$ has yet to be ascertained.

Unlike the schizophrenia spectrum, the autistic spectrum is defined predominantly by the presence of deficits and impairments, rather than by 'positive' symptoms that represent novel patterns of behavior, cognition and affect that are reasonably specific to the condition or its subtypes. A suite of 'positive' symptoms can be described for autism (Table 1), although each of them applies more or less strongly across subsets of autistic individuals. The primary usefulness of 'positive' autistic phenotypes rests in their use for deliniation of discrete sets of causes that convergently mediate autism, and in their ability to facilitate differential diagnosis of autism from other conditions characterized by social and communicative impairments, such as relatively-severe premorbidity to schizophrenia. 
Second, studies of the genetic basis of schizophrenia in relation to schizotypy, the prevalence of autistic and schizotypal traits in non-clinial populations, familial aggregation of phenotypes considered as autistic or schizotypal, quantitative-genetic studies of autistic phenotypes, and studies of genetic variation in specific genes subject to either large-scale or small-scale alterations, provide convergent evidence that schizophrenia-spectrum traits, and autistic-spectrum traits, each grades more or less smoothly from severe impairment into normality ${ }^{98-100}$. In accordance with such continuity, autism and schizophrenia have each been demonstrated to be strongly mediated by effects from de novo and segregating genetic variation of small and large effects, with high heritabilities, but also with high levels of genetic heterogeneity, such that a large number of genetic risk factors, separately and/or in combination, convergently mediates the risk of each condition ${ }^{101-103}$. A primary implication of the continuity between these clinical disorders and normality is that the phenotypes characteristic of schizophrenia and autism spectrum conditions can each be considered as extreme manifestations of normal human variation in cognitive and affective phenotypes ${ }^{73,104,105}$ rather that pathology disembodied from human adaptation.

For both the schizophrenia and autism spectrum, the neurocognitive adaptations most relevant to the disorders are components of the human 'social brain' - the integrated, distributed set of neural systems that subserves the acquisition, processing and deployment of social information ${ }^{44,106-108}$ (Figure 1). To the extent that these psychiatric conditions are mediated by dysregulated 
development and function of the social brain, understanding the ontogeny, modularity, integrated ultimate and proximate functions, and genetic bases of social-brain components becomes fundamental to dissecting the etiologies of autism and schizophrenia, and ultimately, to defining disorders of human development based on biological criteria. A simple, testable model for conceptualizing the adaptive significance of social-brain phenotypes, in relation to their maladaptive dysregulation in schizophrenia spectrum conditions, is provided in Figure 2. This model can be tested and refined by evaluating the normal adaptive functions of specific cognitive-affective phenotypes ${ }^{109}$ in relation to their forms of dysregulation in more or less severe psychiatric conditions, and by systematically elucidating the cognitive-affective correlates of validated schizophrenia or autism 'risk' alleles in non-clinical populations ${ }^{110,111}$.

Despite the continuity of the autism spectrum and the schizophrenia spectrum with normality, the multidimensional distributions of autistic and schizophrenic-spectrum phenotypes in populations of clinical and non-clinical individuals considered together remain unclear, in part because they are some function of the spectrum of salient genetic and environmental variation present in populations. Thus, if the bulk of variation in such phenotypes is mediated by relatively common, segregating alleles each of small effect, normal distributions are expected - as for any polygenic trait. By contrast, rare, commonly de novo mutations of large effect, such as highly-penetrant copy number variants and monogenic causes of autism, should tend to produce discontinuities, with 'spikes' in the distributions at the clinical ends of the spectra and their components. Each 
type of genetic risk factor has now been well documented, especially for schizophrenia, but the spectrum of effect sizes, and risk allele frequencies, remain to be fully elucidated, with much of the heritability for each set of conditions yet to be discovered ${ }^{112}$.

The confrontation of genetic and genomic data with data on diagnosisrelated phenotypes represents one of the most important ongoing developments in understanding the etiologies of autism and schizophrenia. A useful set of models relating genes to psychiatric conditions and their diagnostic phenotypes is described by Fanous and Kendler ${ }^{70}$ : allelic variation may affect risk of the condition itself, risk of some subset of clinical features of the condition, or expression of clinical features but not risk. Genes 'for' autism or schizophrenia ${ }^{68}$ have thus far been evaluated in genome-wide association, copy number variant, and deep-sequencing studies predominantly under models of causation that involve genetic factors mediating risk of the full diagnoses as threshold traits, rather than mediating phenotypic expression, qualitative or quantitative, of the component phenotypes. To the extent that diagnostic phenotypes are genetically as well as phenotypically dissociable, and to the extent that neurodevelopmental disorders are mediated by sets of distinct genetic causes that convergently yield particular diagnostic phenotypes, this approach will be notably inefficient. Much of the heritability of schizophrenia and autism may thus be presently 'missing' due to the phenotypic and genetic structures of the disorders, and not the limitations of genomic technology. Despite such considerations, the observation of a proportion of cases of autism or schizophrenia that appear attributable to 
losses of function or dosage alterations in single genes (e. g. AUTS2, FMR1, DISC1, and NRXN1, among others) indicates that single genes may pleiotropically mediate expression of the full suite of symptoms sufficient for diagnosis, perhaps due to extensive pleiotropy ${ }^{113}$. Such data provide evidence for coherence of autism and schizophrenia as biologically-based conditions, with shared causal linkages between their components.

The primary question that follows from these considerations is whether or not the phenotypic and genetic structures of the autism and schizophrenia spectra - their partially-dissociable phenotypes, continuity with normality, highlyheritable nature, and notable clinical and genetic heterogeneity - can be reconciled such that the two conditions are causally and functionally related in some manner that can be evaluated statistically. If so, then the criteria for defining the two sets of conditions can, in principle, be grounded within a common non-arbitrary, biologically-based, non-historical, yet pragmatic causal framework. Addressing this question involves consideration of explicit alternative models for how the schizophrenia spectrum and autism spectrum are related to one another, and how strong inference can be used to evaluate which model or models best fits the available data.

\section{Alternative models for the relationship of schizophrenia with autism}

Differentiating between alternative models for the relationships of major human psychiatric conditions with one another has important implications for diagnoses, pharmacological and psychological treatment, and strategies for dissection of 
etiology at all levels from genes to psychology. Recent studies ${ }^{114-117}$ have demonstrated how genetic data can be deployed to evaluate explicit alternative hypotheses for the relationship of schizophrenia with bipolar disorder, originally described as a dichotomy in work that followed from the pioneering studies of Emil Kraepelin but now viewed in terms of partial overlap, based on the presence of shared genetic risk factors mediating shared phenotypes. Such analyses have only recently become feasible with the discovery of robustly replicated genetic risk factors, and they allow objective criteria to be applied to nosological frameworks that have thus far been difficult to evaluate rigorously.

Studies by Craddock and colleagues ${ }^{114-117}$ have provided strong evidence for continuity between schizophrenia and bipolar disorder, because specific genetic risk factors - both highly-penetrant alterations and common polymorphisms of small effect, predispose to either one or both of the conditions. As a result, despite the common presence of patients with 'pure' psychotic or mood symptoms, schizophrenia and bipolar disorder grade together phenotypically, apparently due to a continuum of shared and separate genetic risk factors. Such structure should, eventually, allow clinical heterogeneity to be connected with, and mapped onto, genetic heterogeneity, in the additional context of environmentally-based risk.

The relationship of schizophrenia with autism remains much less clear $^{44,62,118-120}$. For some years, Kanner ${ }^{121}$ considered autism as a distinct, early-expressed subtype of schizophrenia, similar in general form to negativesymptom schizophrenia. As noted above, this viewpoint persisted for several 
decades in the context of autism as a manifestation of childhood-onset schizophrenia, and as a disorder characterized predominantly by social deficits similar to those found in negative-symptom schizophrenics ${ }^{122}$. Under this model (Figure 3a), autism should be mediated by a subset of negative-symptom schizophrenia genetic-risk factors - presumably those with early onset or high severity.

Kanner ${ }^{123}$ later renounced the idea of autism as a subtype of schizophrenia, in favor of a view, also supported by Rutter ${ }^{7,8}$, with the conditions as distinct, separate and unrelated to one another (Figure 3b). By this model, autism and schizophrenia should not overlap in genetic risk factors, unless some risk alleles are sufficiently general to underlie a broad swath of cognitive-affective dysfunctions.

Under each of these two hypotheses, autism and schizophrenia each grades independently into normality. Under a third model (Figure 3c), schizophrenia and autism have been considered as diametric (opposite) sets of conditions along a spectrum of social-brain phenotypes, from hypo-development in autism, to normality, to hyper-development in schizophrenia ${ }^{44,124}$. The idea of diametric disorders is novel to psychiatric classification, but can be conceptualized as analogous to neurodevelopmental pathways that can be perturbed in two opposite directions, or signaling pathways can become underversus over-activated. Schizophrenia spectrum conditions may thus develop in part due to losses of function in negative, homeostatic regulators of socialcognitive-affective phenotypes (phenotypes that in autism never matured), which 
leads to differential deficits in social skills that may appear similar to those in autism but develop by fundamentally-different means (Figure 4). Examples of such losses in negative regulation may include over-activity of the mid-brain dopaminergic systems leading to inappropriate assignment of motivational salience to irrelevant objects, people and actions ${ }^{125}$, 'jumping to conclusions' as a risk factor for the formation of delusions ${ }^{126}$ and impairments of corollary discharge systems mediating aspects of hallucinations and passivity phenomenon ${ }^{127,128}$. In a developmental context, social skills are impaired during childhood in autistics due to hypo-development of social brain phenotypes, and, in some cases, childhood social skills are also reduced in individuals premorbid for schizophrenia, though for other reasons.

Diametric etiology of the autism and psychotic-affective spectra, in the context of the normally-developing social brain, takes account of their fractionable natures in that corresponding hypo- and hyper-developed socialbrain phenotypes can be described for the two conditions, each of which may be underlain by some subset of the genetic risk factors that underly the full spectra. Each phenotype of the autism and psychotic-affective spectra can also be directly related to normal social-brain functions that became highly-developed along the human lineage (Figure 5), such that expression of these psychiatric phenotypes becomes explicable in terms of the recent expansion and elaboration of the human social brain ${ }^{44,107,108}$. By contrast, other models of autism in relation to the psychotic-affective spectrum remain disconnected from human evolution and the cognitive-affective adaptations that characterize our lineage. 
By a fourth model, autism overlaps broadly yet partially with schizophrenia, sharing some risk factors and phenotypes but not others (Figure 3d). This model has been motivated by recent genetic evidence for shared loci and pathways mediating both autism and schizophrenia risk ${ }^{129,130}$, as well as by work describing social deficits as central to both Kanner's autism and the 'autistic' symptoms of Blueler's schizophrenia, as described above. Under this hypothesis, autism and schizophrenia are expected to share a subset of risk alleles, which underlie shared social-deficit phenotypes, but each condition should also be mediated by unshared risk alleles that mediate unique phenotypes.

Finally, Craddock and Owen ${ }^{117}$ have proposed a variant of the overlapping model, under which schizophrenia overlaps partially with autism along a spectrum of increasing cognitive impairment, with autism grading into mental retardation (Figure 3e). By this hypothesis, autism and schizophrenia should be mediated by a partially-overlapping set of genetic risk factors, with autism the result of more-severe alterations to the genetic bases of neurodevelopment. This model can help to explain evidence for: (1) high comorbidity of autism with intellectual disability; (2) the presence of cognitive impairments but not mooddisorder symptoms in schizophrenia, autism and intellectual disability; and (3) data showing overlap of risk loci between schizophrenia and autism, as well as between autism and intellectual disability $62,73,131,132$.

The primary assumptions involved in using strong inference to evaluate the predictions of these alternative hypotheses include: (1) the absence of 
ascertainment biases, which have been proposed to explain reports of relatively low IQ in autism spectrum conditions, given that lower-IQ individuals are more likely to be brought to the attention of clinicians ${ }^{84,85,133}$; (2) accuracy of diagnoses, especially with regard to 'false positive' diagnoses of relatively-severe premorbidity to schizophrenia as autism spectrum, as described above; (3) validity of the associations between genetic risk factors and diagnosed conditions, especially for autism, which has been subject to considerably less well-replicated, large-sample genetic study than schizophrenia; and (4) the possibility that the hypotheses are not mutually exclusive, due to the presence of genetic variants and polymorphisms that increase risk of any psychiatric condition non-specifically, or due to relationships between autism and schizophrenia that are more complex than the models depicted here. These assumptions are unlikely to be met, but each can be addressed with regard to future studies and the nature of the potential biases and inaccuracies that they introduce.

Crespi et al. ${ }^{62}$ used copy number variant and genetic association data to evaluate the former four hypotheses described above. They reported evidence of statistically significant increased sharing of risk genes, from genetic association studies, between autism and schizophrenia. This sharing of risk genes was generated by two patterns in the relationships of alleles and genotypes to the conditions: (a) for some loci, the same allele mediated risk of both conditions; and (b) for other loci, autism was associated with one allele or genotype and schizophrenia was associated with the other; other loci could not 
be directly compared due to heterogeneity of markers or complexity of the findings.

For copy number variant data, Crespi et al. ${ }^{62}$ found diametric patterns at three $(16 p 11.2,22 q 11.2,22 q 13.3)$ of the seven copy number variant loci with sufficient data for analysis: deletions were statistically associated with one condition from case-control studies, and duplications were statistically associated with the other. By contrast, one of the loci (16p13.1) showed duplications associated with both conditions, and for 1q21.1, deletions were associated with both autism and schizophrenia (though with only two autism cases reported, compared to 15 cases for schizophrenia), whereas duplications were associated only with autism.

Taken together, these results from genetic-association and copy-number variant data can be interpreted as apparent falsification of the hypotheses that autism is subsumed within schizophrenia (Figure 3a), or that schizophrenia and autism are genetically independent of one another (Figure 3b). The findings of diametric patterns for several copy number variant loci, and some geneticassociation loci, are compatible with the diametric model (Figure 3c) but the presence of some unambiguously-shared risk factors support a model of partially-shared risk and some degree of overlap, or a substantial degree of falsepositive diagnoses of premorbidity to schizophrenia as autism (or negativesymptom schizophrenia and schizotypy as autism). These questions require further, focused study in hypothesis-testing frameworks, to assess the prevalence of false positives, and jointly evaluate the genetic underpinnings of 
autism and schizophrenia using genome-wide approaches with the same suites of markers.

One possible reconciliation of diametric and overlap models is that some set of loci exerts diametric genetic effects, while at other loci, some alleles mediate mild impairments of social cognition that increase risk of both autism and schizophrenia. For example, in autism such alleles may contribute to hypodevelopment of social brain phenotypes, whereas in schizophrenia they increase the likelihood of social-brain dysregulation by other means such as deficits in theory of mind, after the social brain has developed to maturity. Alleles with relatively general effects on social-cognitive development, which would be analogous to intellectual-disability genes but differentially affect social cognition, should also contribute to premorbid phenotypes of schizophrenia involving impairments in sociality and language.

Ultimately, robust evaluation of alternative hypotheses for the relationship of autism with schizophrenia will require in-depth analyses of the neurodevelopmental and neuronal-function effects of different alterations to the genes that mediate social-brain function and dysfunction, and integrative data from diverse disciplines other than genetics, especially the neurosciences and psychology. The primary impediments to further testing of the models are likely to be reified conceptualizations of autism and the schizophrenia spectrum divorced from social brain adaptations, and perspectives from psychology that focus on deficits rather than their causes. Indeed, substantive progress in understanding these neurodevelopmental conditions is especially likely to come from analyses 
that span and connect levels of analysis, from genes to intermediate neurological and behavioral phenotypes, and from such phenotypes to psychiatric conditions $^{117,134-137}$. Head size provides a paradigmatic case in point, as relatively large head size or brain size have been reported in idiopathic autism, syndromic autism, and autism associated with specific copy number variants, and causes of large head size in autism have been ascertained in terms of growth-signaling pathway dysregulation ${ }^{44,62}$. Large head size has also been demonstrated to mediate enhanced local compared to global information processing $^{138}$, a phenotype characteristic of autism that may connect directly to social and non-social impairments as well as explain enhancements of some sensory and cognitive skills ${ }^{81}$.

An important practical implication of understanding the relationship between schizophrenia and autism is its potential usefulness in developing pharmacological therapies based on similar, independent, or diametric alterations to neurological systems. For example, antagonists of mGLUR5 signaling have recently been deployed for treatment of the autistic syndrome Fragile $X^{139}$, while agonists of this pathway are under development for schizophrenia ${ }^{140}$; similar considerations may apply to treatments for autism and schizophrenia that target the cholinergic system ${ }^{141,142}$. The presence and nature of similarities and differences between autism and schizophrenia may thus reciprocally illuminate the causes and potential treatments for both conditions, and motivate psychiatric classifications and associated therapies based on biology rather than symptoms $^{143}$. 


\section{Conclusions and future directions}

Recent accelerating progress in genetics, molecular biology, and neuroscience have brought psychiatry into a new era, facing the first realistic hopes of comprehensively characterizing the genetic underpinnings of autism and schizophrenia spectrum conditions and developing rational therapies based on alterations to sets of specific biological pathways ${ }^{144}$. Psychiatric classification, and psychological methods based on categorizing and correlating deficits, have lagged behind such advances, and indeed may impede progress to the extent that they conflate disparate conditions, combine individuals across highlyfractionable DSM diagnoses, deny the reality and effects of false-positive diagnoses, ignore the adaptive significance of the cognitive-affective mechanisms disrupted by autism and schizophrenia spectrum phenotypes, and echo Blueler's century-old conceptualization of autism. Indeed, until interdisciplinary studies that integrate across levels from genes to neurodevelopment to psychological phenotypes are systematically deployed, in hypothesis-testing frameworks that use strong inference ${ }^{62,145}$, we should expect to remain, as penned by García Márquez, in "permanent alternation between excitement and disappointment, doubt and revelation, to such an extreme that no one knows for certain where the limits of reality lay" - for yet another 100 years. 


\section{Acknowledgements}

I am grateful to NSERC for financial support, to C. Badcock and N. Craddock for discussions, and to M. Ritsner for inviting me to write this review.

\section{References}

1. Bleuler, PE. Dementia Praecox or the Group of Schizophrenias. Trans. by Joseph Zinkin. 1951; Allen and Unwin, London

2. Kanner L. Autistic disturbances of affective contact. Nerv Child $1943 ; 2: 217-250$

3. McNally K. Eugene Bleuler's four As. Hist Psychol 2009;12:43-59

4. Remschmidy HE, Schulz E, Martin M, et al. Childhood-onset schizophrenia: history of the concept and recent studies. Schizophr Bull 1994;20:727-745

5. Starling J, Dossetor D. Pervasive developmental disorders and psychosis. Curr Psychiatry Rep 2009;11:190-196

6. Kolvin I. Studies in the childhood psychoses. I. Diagnostic criteria and classification. Br J Psychiatry 1971;118:381-384

7. Rutter M. Concepts of autism: A review of research. J Child Psychol Psychiatry 1968;9:1-25

8. Rutter M. Childhood schizophrenia reconsidered. J Autism Child Schizophr 1972;2:315-337

9. Rutter M. Diagnosis and definition of childhood autism. J Autism Child Schizophr 1978;8:139-161 
10. Wing L. The definition and prevalence of autism: a review. Eur Child Adolesc Psych 1993;2:61-74

11. Lauritsen MB, Pedersen CB, Mortensen PB. The incidence and prevalence of pervasive developmental disorders: a Danish population-based study. Psychol Med 2004;34:1339-1346

12. Happé $F$, Ronald A. The 'fractionable autism triad': a review of evidence from behavioural, genetic, cognitive and neural research. Neuropsychol Rev 2008;18:287-304

13. Walker DR, Thompson A, Zwaigenbaum L, et al. Specifying PDD-NOS: a comparison of PDD-NOS, Asperger syndrome, and autism. J Am Acad Child Adolesc Psychiatry 2004;43:172-180

14. Brüne M. "Theory of mind" in schizophrenia: a review of the literature. Schizophr Bull 2005;31:21-42

15. Covington MA, He C, Brown C. et al. Schizophrenia and the structure of language: the linguist's view. Schizophr Res 2005;77:85-98

16. McKenna, P. and Oh, T. Schizophrenic Speech: Making Sense of Bathroots and Ponds that Fall in Doorways. 2005; Cambridge University Press, New York.

17. Bora E, Yücel M, Pantelis C. Theory of mind impairment: a distinct traitmarker for schizophrenia spectrum disorders and bipolar disorder? Acta Psychiatr Scand 2009;120:253-264

18. Fett AK, Viechtbauer W, Dominguez MD, et al. The relationship between neurocognition and social cognition with functional outcomes in 
schizophrenia: A meta-analysis. Neurosci Biobehav Rev. 2010 in press

19. Cunill R, Castells X, Simeon D. Relationships between obsessivecompulsive symptomatology and severity of psychosis in schizophrenia: a systematic review and meta-analysis. J Clin Psychiatry 2009;70:70-82

20. Jacob S, Landeros-Weisenberger A, Leckman JF. Autism spectrum and obsessive-compulsive disorders: OC behaviors, phenotypes and genetics. Autism Res 2009;2:293-311

21. Konstantareas MM, Hewitt T. Autistic disorder and schizophrenia: diagnostic overlaps. J Autism Dev Disord 2001;31:19-28

22. Sheitman BB, Kraus JE, Bodfish JW, et al. Are the negative symptoms of schizophrenia consistent with an autistic spectrum illness? Schizophr Res 2004;69:119-120

23. Hurst RM, Nelson-Gray RO, Mitchell JT, et al. The relationship of Asperger's characteristics and schizotypal personality traits in a nonclinical adult sample. J Autism Dev Disord 2007;37:1711-1720

24. Esterberg ML, Trotman HD, Brasfield JL, et al. Childhood and current autistic features in adolescents with schizotypal personality disorder. Schizophr Res 2008;104:265-273

25. Couture SM, Penn DL, Losh M, et al. Comparison of social cognitive functioning in schizophrenia and high functioning autism: more convergence than divergence. Psychol Med 2010;40:569-579 
26. Roy MA, Maziade M, Labbé A, et al. Male gender is associated with deficit schizophrenia: a meta-analysis. Schizophr Res 2001;47:141-147

27. Sporn AL, Addington AM, Gogtay N, et al. Pervasive developmental disorder and childhood-onset schizophrenia: comorbid disorder or a phenotypic variant of a very early onset illness? Biol Psychiatry 2004;55:989-994

28. Reaven JA, Hepburn SL, Ross RG. Use of the ADOS and ADI-R in children with psychosis: importance of clinical judgment. Clin Child Psychol Psychiatry 2008;13:81-94

29. Hurley RS, Losh M, Parlier M, et al. The broad autism phenotype questionnaire. J Autism Dev Disord 2007;37:1679-1690

30. Venables, PH, Wilkins S, Mitchell, DA, et al. A scale for the measurement of schizotypy. Pers Individ Diff 1990;11:481-495

31. Raine A, Benishay D. The SPQ-B: A brief screening instrument for schizotypal personality disorder. J Pers Disord 1995;9:346-355

32. Wuthrich VM, Bates TC. Confirmatory factor analysis of the three-factor structure of the schizotypal personality questionnaire and Chapman schizotypy scales. J Pers Assess 2006;87:292-304

33. Sugihara G, Tsuchiya KJ, Takei N. Distinguishing broad autism phenotype from schizophrenia-spectrum disorders. J Autism Dev Disord 2008;38:1998-1999 
34. Baker KD, Skuse DH. Adolescents and young adults with 22q11 deletion syndrome: psychopathology in an at-risk group. Br J Psychiatry 2005;186:115-120

35. DeLisi LE, Maurizio AM, Svetina C, et al. Klinefelter's syndrome (XXY) as a genetic model for psychotic disorders. Am J Med Genet B Neuropsychiatr Genet 2005;135B:15-23

36. Antshel KM, Aneja A, Strunge L, et al. Autistic spectrum disorders in velocardio facial syndrome (22q11.2 deletion). J Autism Dev Disord 2007;37:1776-1786.

37. Dimitropoulos A, Schultz RT. Autistic-like symptomatology in Prader-Willi syndrome: a review of recent findings. Curr Psychiatry Rep 2007;9:159-164

38. Jha P, Sheth D, Ghaziuddin M. Autism spectrum disorder and Klinefelter syndrome. Eur Child Adolesc Psychiatry 2007;16:305-308

39. van Rijn S, Swaab H, Aleman A, et al. Social behavior and autism traits in a sex chromosomal disorder: Klinefelter (47XXY) syndrome. J Autism Dev Disord 2008;38:1634-1641

40. Soni S, Whittington J, Holland AJ, et al. The phenomenology and diagnosis of psychiatric illness in people with Prader-Willi syndrome. Psychol Med 2008;38:1505-1514

41. Webb T, Maina EN, Soni S, et al. In search of the psychosis gene in people with Prader-Willi syndrome. Am J Med Genet A 2008;146:843853 
42. Bruining $\mathrm{H}$, de Sonneville $\mathrm{L}$, Swaab $\mathrm{H}$, et al. Dissecting the clinical heterogeneity of autism spectrum disorders through defined genotypes. PLoS One 2010;5:e10887

43. Karayiorgou M, Simon TJ, Gogos JA. 22q11.2 microdeletions: linking DNA structural variation to brain dysfunction and schizophrenia. Nat Rev Neurosci 2010;11:402-416

44. Crespi B, Badcock C. Psychosis and autism as diametrical disorders of the social brain. Behav Brain Sci 2008;31:241-261; discussion 261320

45. Crespi B, Summers K, Dorus S. Genomic sister-disorders of neurodevelopment: an evolutionary approach. Evol Appl 2009;2:81100

46. Jones $\mathrm{P}$. The early origins of schizophrenia. Br Med Bull 1997;53:135-155

47. Sobin C, Blundell ML, Conry A, et al. Early, non-psychotic deviant behavior in schizophrenia: a possible endophenotypic marker for genetic studies. Psychiatry Res 2001;101:101-113

48. Schenkel LS, Silverstein SM. Dimensions of premorbid functioning in schizophrenia: a review of neuromotor, cognitive, social, and behavioral domains. Genet Soc Gen Psychol Monogr 2004;130:241270

49. Eliez S. Autism in children with 22q11.2 deletion syndrome. J Am Acad Child Adolesc Psychiatry 2007;46:433-434 
50. Monte RC, Goulding SM, Compton MT. Premorbid functioning of patients with first-episode nonaffective psychosis: a comparison of deterioration in academic and social performance, and clinical correlates of Premorbid Adjustment Scale scores. Schizophr Res $2008 ; 104: 206-213$

51. Tarbox SI, Pogue-Geile MF. Development of social functioning in preschizophrenia children and adolescents: a systematic review. Psychol Bull 2008;134:561-583

52. Saracco-Alvarez R, Rodríguez-Verdugo S, García-Anaya M, et al. Premorbid adjustment in schizophrenia and schizoaffective disorder. Psychiatry Res 2009;165:234-240

53. Tandon R, Nasrallah HA, Keshavan MS. Schizophrenia, "just the facts" 4. Clinical features and conceptualization. Schizophr Res 2009;110:1-23

54. Keshavan MS, Kulkarni S, Bhojraj T, et al. Premorbid cognitive deficits in young relatives of schizophrenia patients. Front Hum Neurosci 2010;3:62

55. Feinstein C, Singh S. Social phenotypes in neurogenetic syndromes. Child Adolesc Psychiatr Clin N Am 2007;16:631-647

56. Shinawi M, Liu P, Kang SH, et al.Recurrent reciprocal 16p11.2 rearrangements associated with global developmental delay, behavioural problems, dysmorphism, epilepsy, and abnormal head size. J Med Genet 2010;47:332-341

57. Rapoport J, Chavez A, Greenstein D, et al. Autism spectrum disorders 
and childhood-onset schizophrenia: clinical and biological contributions to a relation revisited. J Am Acad Child Adolesc Psychiatry 2009;48:10-18

58. Weiss LA, Shen Y, Korn JM, et al. Association between microdeletion and microduplication at 16p11.2 and autism. N Engl J Med 2008;358:667675

59. Ben-Shachar S, Lanpher B, German JR, et al. Microdeletion 15q13.3: a locus with incomplete penetrance for autism, mental retardation, and psychiatric disorders. J Med Genet 2009;46:382-388

60. Doornbos M, Sikkema-Raddatz B, Ruijvenkamp CA, et al. Nine patients with a microdeletion $15 q 11.2$ between breakpoints 1 and 2 of the Prader-Willi critical region, possibly associated with behavioural disturbances. Eur J Med Genet 2009;52:108-115

61. McCarthy SE, Makarov V, Kirov G, et al. Microduplications of 16p11.2 are associated with schizophrenia. Nat Genet 2009;41:1223-1227

62. Crespi B, Stead P, Elliot M. Comparative genomics of autism and schizophrenia. Proc Natl Acad Sci U S A 2010;107 Suppl 1:1736-1741

63. Kumar RA, KaraMohamed S, Sudi J, et al. Recurrent 16p11.2 microdeletions in autism. Hum Mol Genet 2008;17:628-638

64. Glessner JT, Wang K, Cai G, et al. Autism genome-wide copy number variation reveals ubiquitin and neuronal genes. Nature 2009;459:569573 
65. de Bruin El, de Nijs PF, Verheij F, et al. Multiple complex developmental disorder delineated from PDD-NOS. J Autism Dev Disord $2007 ; 37: 1181-1191$

66. Sprong M, Becker HE, Schothorst PF, et al. Pathways to psychosis: a comparison of the pervasive developmental disorder subtype Multiple Complex Developmental Disorder and the "At Risk Mental State". Schizophr Res 2008;99:38-47

67. Gothelf D, Frisch A, Michaelovsky E, et al. Velo-Cardio-Facial Syndrome. J Ment Health Res Intellect Disabil 2009;2:149-167

68. Kendler KS. "A gene for...": the nature of gene action in psychiatric disorders. Am J Psychiatry 2005;162:1243-1252

69. Blackwood DH, Pickard BJ, Thomson PA, et al. Are some genetic risk factors common to schizophrenia, bipolar disorder and depression? Evidence from DISC1, GRIK4 and NRG1. Neurotox Res 2007;11:7383

70. Fanous AH, Kendler KS. Genetics of clinical features and subtypes of schizophrenia: a review of the recent literature. Curr Psychiatry Rep 2008;10:164-170

71. Muir WJ, Pickard BS, Blackwood DH. Disrupted-in-Schizophrenia-1. Curr Psychiatry Rep 2008;10:140-147

72. Walters JT, Corvin A, Owen MJ, et al. Psychosis susceptibility gene ZNF804A and cognitive performance in schizophrenia. Arch Gen Psychiatry 2010;67:692-700 
73. Van Os J. Are psychiatric diagnoses of psychosis scientific and useful? The case of schizophrenia. J Ment Health 2010;19:305-317

74. Linney YM, Murray RM, Peters ER, et al. A quantitative genetic analysis of schizotypal personality traits. Psychol Med 2003;33:803-816

75. Schürhoff F, Laguerre A, Szöke A, et al. Schizotypal dimensions: continuity between schizophrenia and bipolar disorders. Schizophr Res 2005;80:235-242

76. Happé F, Ronald A, Plomin R. Time to give up on a single explanation for autism. Nat Neurosci 2006;9:1218-20

77. Ronald $\mathrm{A}$, Larsson $\mathrm{H}$, Anckarsäter $\mathrm{H}$, et al. A twin study of autism symptoms in Sweden. Mol Psychiatry 2010 in press

78. Mandy WP, Skuse DH. What is the association between the socialcommunication element of autism and repetitive interests, behaviours and activities? J Child Psychol Psychiatry 2008;49:795-808

79. Soulières I, Dawson M, Samson F, et al. Enhanced visual processing contributes to matrix reasoning in autism. Hum Brain Mapp $2009 ; 30: 4082-4107$

80. Russell-Smith SN, Maybery MT, Bayliss DM. Are the autism and positive schizotypy spectra diametrically opposed in local versus global processing? J Autism Dev Disord 2010;40:968-977

81. Mottron L, Dawson M, Soulières I. Enhanced perception in savant syndrome: patterns, structure and creativity. Philos Trans R Soc Lond B Biol Sci 2009;364:1385-1391 
82. Happé F, Vital P. What aspects of autism predispose to talent? Philos Trans R Soc Lond B Biol Sci 2009;364:1369-1375

83. Newman TM, Macomber D, Naples AJ, et al. Hyperlexia in children with autism spectrum disorders. J Autism Dev Disord 2007;37:760-774

84. Dawson M, Soulières I, Gernsbacher MA, et al. The level and nature of autistic intelligence. Psychol Sci 2007;18:657-662

85. Hayashi M, Kato M, Igarashi K, et al. Superior fluid intelligence in children with Asperger's disorder. Brain Cogn 2008;66:306-310

86. Prior M, Perry D, Gajzago C. Kanner's syndrome or early-onset psychosis: a taxonomic analysis of 142 cases. J Autism Child Schizophr 1975;5:71-80

87. Cunningham AB, Schreibman L. Stereotypy in autism: the importance of function. Res Autism Spectr Disord 2008;2:469-479

88. Goldman S, Wang C, Salgado MW, et al. Motor stereotypies in children with autism and other developmental disorders. Dev Med Child Neurol $2009 ; 51: 30-38$

89. Bonnel A, McAdams S, Smith B, et al. Enhanced pure-tone pitch discrimination among persons with autism but not Asperger syndrome. Neuropsychologia 2010;48:2465-2475

90. Ashwin E, Ashwin C, Rhydderch D, et al. Eagle-eyed visual acuity: an experimental investigation of enhanced perception in autism. Biol Psychiatry 2009;65:17-21 
91. Mottron L, Dawson M, Soulières I, et al. Enhanced perceptual functioning in autism: an update, and eight principles of autistic perception. J Autism Dev Disord 2006;36:27-43

92. Scott MM, Deneris ES. Making and breaking serotonin neurons and autism. Int J Dev Neurosci 2005;23:277-285

93. Whitaker-Azmitia PM. Behavioral and cellular consequences of increasing serotonergic activity during brain development: a role in autism? Int $\mathrm{J}$ Dev Neurosci 2005;23:75-83

94. Mraz KD, Dixon J, Dumont-Mathieu T, et al. Accelerated head and body growth in infants later diagnosed with autism spectrum disorders: a comparative study of optimal outcome children. J Child Neurol 2009;24:833-845

95. Redcay E, Courchesne E. When is the brain enlarged in autism? A metaanalysis of all brain size reports. Biol Psychiatry 2005;58:1-9

96. Herbert MR, Ziegler DA, Deutsch CK, et al. Dissociations of cerebral cortex, subcortical and cerebral white matter volumes in autistic boys. Brain 2003;126:1182-1192

97. Bigler ED, Abildskov TJ, Petrie JA, et al. Volumetric and voxel-based morphometry findings in autism subjects with and without macrocephaly. Dev Neuropsychol 2010;35:278-295

98. Fanous A, Gardner C, Walsh D, et al. Relationship between positive and negative symptoms of schizophrenia and schizotypal symptoms in nonpsychotic relatives. Arch Gen Psychiatry 2001;58:669-673 
99. Skuse DH, Mandy WP, Scourfield J. Measuring autistic traits: heritability, reliability and validity of the Social and Communication Disorders Checklist. Br J Psychiatry 2005;187:568-572

100. Fanous $\mathrm{AH}$, Neale MC, Gardner CO, et al. Significant correlation in linkage signals from genome-wide scans of schizophrenia and schizotypy. Mol Psychiatry 2007;12:958-965

101. Abrahams BS, Geschwind DH. Advances in autism genetics: on the threshold of a new neurobiology. Nat Rev Genet 2008;9:341-355

102. Weiss LA. Autism genetics: emerging data from genome-wide copynumber and single nucleotide polymorphism scans. Expert Rev Mol Diagn 2009;9:795-803

103. Owen MJ, Craddock N, O'Donovan MC. Suggestion of roles for both common and rare risk variants in genome-wide studies of schizophrenia. Arch Gen Psychiatry 2010;67:667-673

104. Nesse RM. Cliff-edged fitness functions and the persistence of schizophrenia. Behav Brain Sci 2004;27:862-863

105. Jung RE, Grazioplene R, Caprihan A, et al. White matter integrity, creativity, and psychopathology: disentangling constructs with diffusion tensor imaging. PLoS One 2010;5:e9818

106. Brüne M, Brüne-Cohrs U. Theory of mind--evolution, ontogeny, brain mechanisms and psychopathology. Neurosci Biobehav Rev $2006 ; 30: 437-455$ 
107. Burns JK. Psychosis: a costly by-product of social brain evolution in Homo sapiens. Prog Neuropsychopharmacol Biol Psychiatry 2006;30:797814

108. Frith CD. Social cognition. Philos Trans R Soc Lond B Biol Sci 2008;363:2033-2039

109. Keller MC, Nesse RM. The evolutionary significance of depressive symptoms: different adverse situations lead to different depressive symptom patterns. J Pers Soc Psychol 2006;91:316-330

110. Jansen A, Krach S, Krug A, et al. A putative high risk diplotype of the G72 gene is in healthy individuals associated with better performance in working memory functions and altered brain activity in the medial temporal lobe. Neuroimage 2009;45:1002-1008

111. Kircher T, Krug A, Markov V, et al. Genetic variation in the schizophreniarisk gene neuregulin 1 correlates with brain activation and impaired speech production in a verbal fluency task in healthy individuals. Hum Brain Mapp 2009;30:3406-3416

112. Manolio TA, Collins FS, Cox NJ, et al. Finding the missing heritability of complex diseases. Nature 2009;461:747-753

113. Hennah W, Porteous D. The DISC1 pathway modulates expression of neurodevelopmental, synaptogenic and sensory perception genes. PLoS One 2009;4:e4906

114. Craddock, N., O'Donovan, M. C. \& Owen, M. J. The genetics of schizophrenia and bipolar disorder: Dissecting psychosis. J Med 
Genet 2005;42:193-204

115. O'Donovan MC, Craddock NJ, Owen MJ. Genetics of psychosis; insights from views across the genome. Hum Genet 2009;126:3-12

116. Craddock N, O'Donovan MC, Owen MJ. Psychosis genetics: modeling the relationship between schizophrenia, bipolar disorder, and mixed (or "schizoaffective") psychoses. Schizophr Bull 2009;35:482-490

117. Craddock N, Owen MJ. The Kraepelinian dichotomy - going, going... but still not gone. Br J Psychiatry 2010;196:92-95

118. Petty LK, Ornitz EM, Michelman JD, et al. Autistic children who become schizophrenic. Arch Gen Psychiatry 1984;41:129-135

119. Goldstein G, Minshew NJ, Allen DN, et al. High-functioning autism and schizophrenia: a comparison of an early and late onset neurodevelopmental disorder. Arch Clin Neuropsychol 2002;17:461475

120. Solomon M, Ozonoff S, Carter C, et al. Formal thought disorder and the autism spectrum: relationship with symptoms, executive control, and anxiety. J Autism Dev Disord 2008;38:1474-1484

121. Kanner L, Problems of nosology and psychodynamics of early infantile autism. Am J Orthopsychiatry 1949;19:416-426

122. Frith $C D$, Frith U. Elective affinities in schizophrenia and childhood autism. In: P. Bebbington, ed. Social Psychiatry: Theory, Methodology and Practice. Transactions Press; 1991:65-88 
123. Kanner L. Infantile autism and the schizophrenias. Behav Sci $1965 ; 10: 412-420$

124. Badcock C. The Imprinted Brain: How Genes Set the Balance Between Autism and Psychosis. 2009; Jessica Kingsley Publishers, London, UK.

125. Murray GK, Corlett PR, Clark L, et al. Substantia nigra/ventral tegmental reward prediction error disruption in psychosis. Mol Psychiatry $2008 ; 13: 239,267-276$

126. Woodward TS, Munz M, LeClerc C, et al. Change in delusions is associated with change in "jumping to conclusions". Psychiatry Res 2009;170:124-127

127. Ford JM, Roach BJ, Faustman WO, et al. Out-of-synch and out-of-sorts: dysfunction of motor-sensory communication in schizophrenia. Biol Psychiatry 2008;63:736-743

128. Taylor JG. A neural model of the loss of self in schizophrenia. Schizophr Bull 2010 in press

129. Iossifov I, Zheng T, Baron M, et al. Genetic-linkage mapping of complex hereditary disorders to a whole-genome molecular-interaction network. Genome Res 2008;18:1150-1162

130. Burbach JP, van der Zwaag B. Contact in the genetics of autism and schizophrenia. Trends Neurosci 2009;32:69-72

131. Matson JL, Shoemaker M. Intellectual disability and its relationship to autism spectrum disorders. Res Dev Disabil 2009;30:1107-1114 
132. Bora E, Yücel M, Pantelis C. Cognitive impairment in schizophrenia and affective psychoses: implications for DSM-V criteria and beyond. Schizophr Bull 2010;36:36-42

133. Skuse DH. Rethinking the nature of genetic vulnerability to autistic spectrum disorders. Trends Genet 2007;23:387-395

134. Prasad KM, Keshavan MS. Structural cerebral variations as useful endophenotypes in schizophrenia: do they help construct "extended endophenotypes"? Schizophr Bull 2008;34:774-790

135. Tan HY, Callicott JH, Weinberger DR. Intermediate phenotypes in schizophrenia genetics redux: is it a no brainer? Mol Psychiatry $2008 ; 13: 233-238$

136. Allen AJ, Griss ME, Folley BS, et al. Endophenotypes in schizophrenia: a selective review. Schizophr Res 2009;109:24-37

137. Kendler KS, Neale MC. Endophenotype: a conceptual analysis. Mol Psychiatry 2010 in press

138. White S, O'Reilly H, Frith U. Big heads, small details and autism. Neuropsychologia 2009;47:1274-1281

139. Berry-Kravis E, Hessl D, Coffey S, et al. A pilot open label, single dose trial of fenobam in adults with fragile X syndrome. J Med Genet 2009;46:266-271

140. Conn PJ, Lindsley CW, Jones CK. Activation of metabotropic glutamate receptors as a novel approach for the treatment of schizophrenia. Trends Pharmacol Sci 2009;30:25-31 
141. Lippiello PM. Nicotinic cholinergic antagonists: a novel approach for the treatment of autism. Med Hypotheses 2006;66:985-990

142. Olincy A, Harris JG, Johnson LL, et al. Proof-of-concept trial of an alpha7 nicotinic agonist in schizophrenia. Arch Gen Psychiatry 2006;63:630638

143. Kendler KS. An historical framework for psychiatric nosology. Psychol Med 2009;39:1935-1941

144. Ehninger D, Li W, Fox K, et al. Reversing neurodevelopmental disorders in adults. Neuron 2008;60:950-960

145. Cannon TD. What is the role of theories in the study of schizophrenia? Schizophr Bull 2009;35:563-567 
Table 1. Psychological, behavioral, neurological and physiological traits that can be considered as 'positive' symptoms of autism

Trait

Studies

Enhanced local information processing, visual-spatial skills

Savant skills, islets of ability

Hyperlexia

High fluid intelligence

Insistence on sameness

Self-stimulatory behaviors

Enhanced perceptual functions

High serotonin levels

Large head, brain size

Increased white matter
Soulieres et al. ${ }^{79}$; RussellSmith et al. ${ }^{80}$

Mottron et al. ${ }^{81}$; Happé Vital ${ }^{82}$

Newman et al. ${ }^{83}$

Dawson et al. ${ }^{84}$; Hayashi et al. ${ }^{85}$

Kanner ${ }^{2}$; Prior et al. ${ }^{86}$

Cunningham and Schreibman ${ }^{87}$; Goldman et al. ${ }^{88}$

Bonnel et al. ${ }^{89}$; Ashwin et al. ${ }^{90}$; Mottron et al. ${ }^{91}$

Scott and Deneris ${ }^{92}$; WhitakerAzmitia $^{93}$

Mraz et al. ${ }^{94}$; Redcay and Courchesne $^{95}$

Herbert et al ${ }^{96}$; Bigler et al. ${ }^{97}$ 
Figure 1. Functional partitioning of components of the human 'social brain', mainly from imaging and lesion studies. $\mathrm{A}=$ amygdala; $\mathrm{aCC}=$ anterior cingulate cortex; $F G$ = fusiform gyrus; iOG = inferior orbital gyrus; latPC = lateral parietal cortex; $\mathrm{mPFC}=$ medial prefrontal cortex; oPFC $=$ orbital prefrontal cortex; $\mathrm{pCC}=$ posterior cingulate cortex; pIFG = posterior inferior frontal gyrus; $\mathrm{pSTG}=$ posterior superior temporal gyrus; $\mathrm{PT}=$ planum temporale; $\mathrm{STS}=$ superior temporal sulcus. The cerebellum ('C') also plays important roles in social-brain cognition and affect. Neuroanatomical positions are approximate.

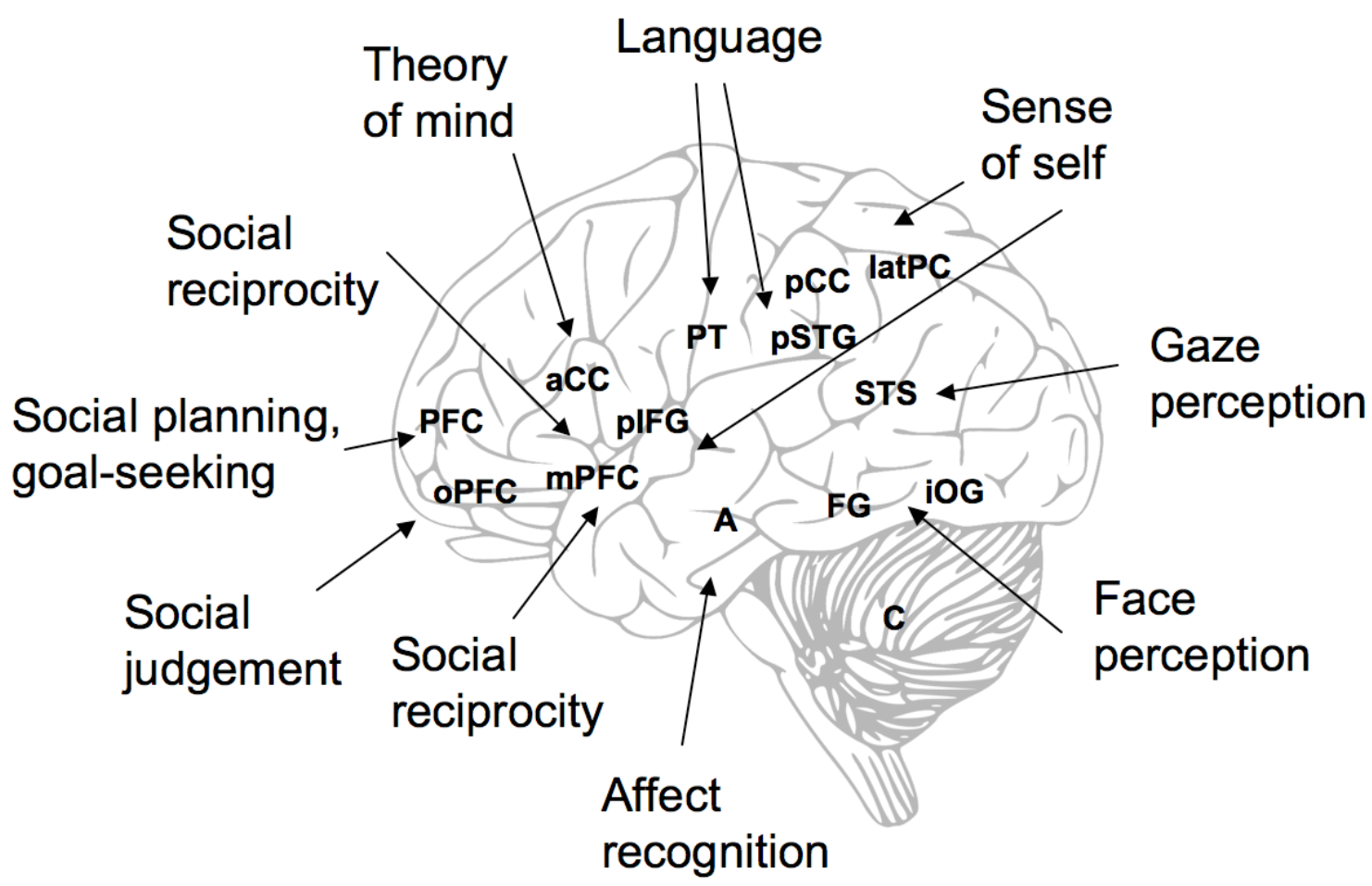


Figure 2. The social, cognitive-affective context of schizophrenia spectrum phenotypes, considered as dysregulated manifestations of neurologicalpsychological traits that are conditionally adaptive.

Conditional cognitive-affective adaptations
Dysregulation of usually-adaptive mechanisms

social-brain cognition

causal, associative thought

schizophrenia

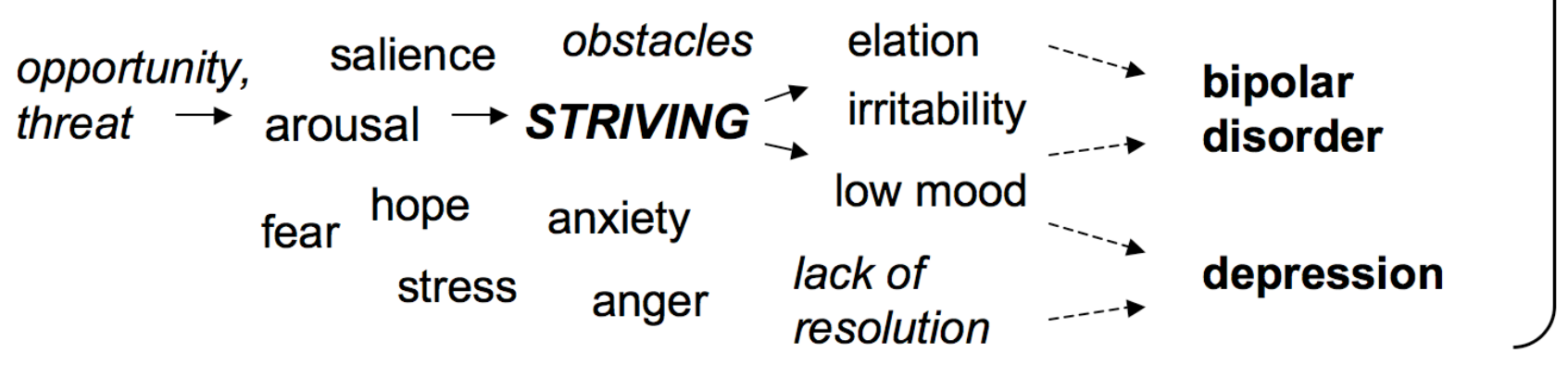

social-brain affect

Reaching goals; new opportunities; giving up on difficult goals 
Figure 3. Alternative models for the relationship of autism with schizophrenia and bipolar disorder, in comparison to normality
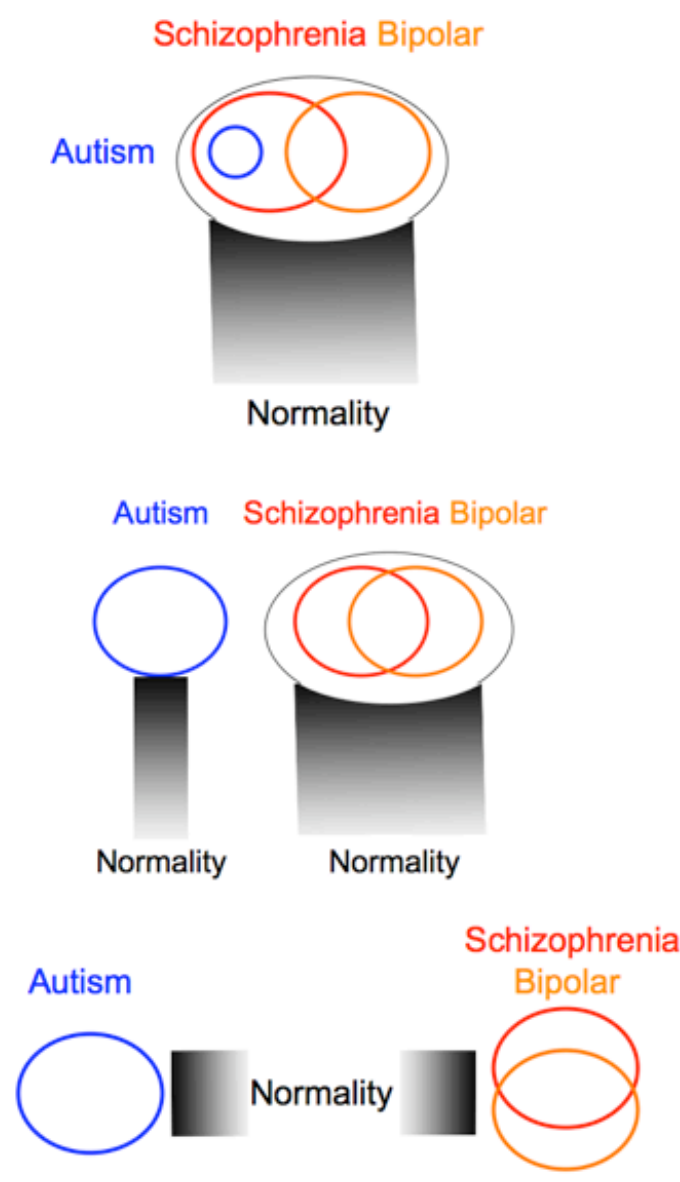

Autism Schizophrenia Bipolar
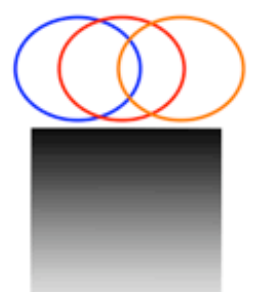

Normality
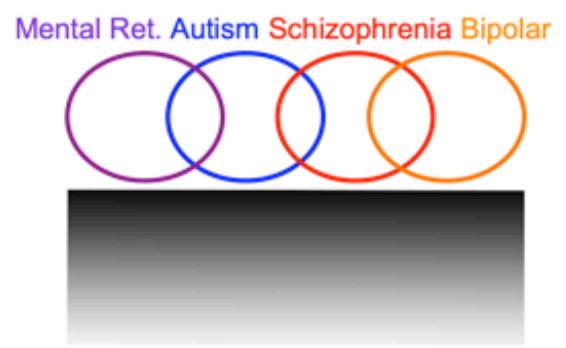

Normality

\section{(a) Subsumed}

(Kanner ${ }^{121}$;

Frith and Frith ${ }^{122}$ )

\author{
(b) Separate \\ (Kanner ${ }^{123}$; \\ Rutter $^{7-9}$; \\ DSM III-V)
}

(d) Overlapping I
(lossifov et al. ${ }^{129}$ )

(d) Overlapping I
(lossifov et al. ${ }^{129}$ )
(c) Diametric
(Crespi and
Badcock $^{44,62}$ )

(e) Overlapping II

(Craddock and Owen ${ }^{117}$ ) 
Figure 4. The development of autism and schizophrenia, with regard to the timing, causes and expression of social-cognitive-affective impairments.

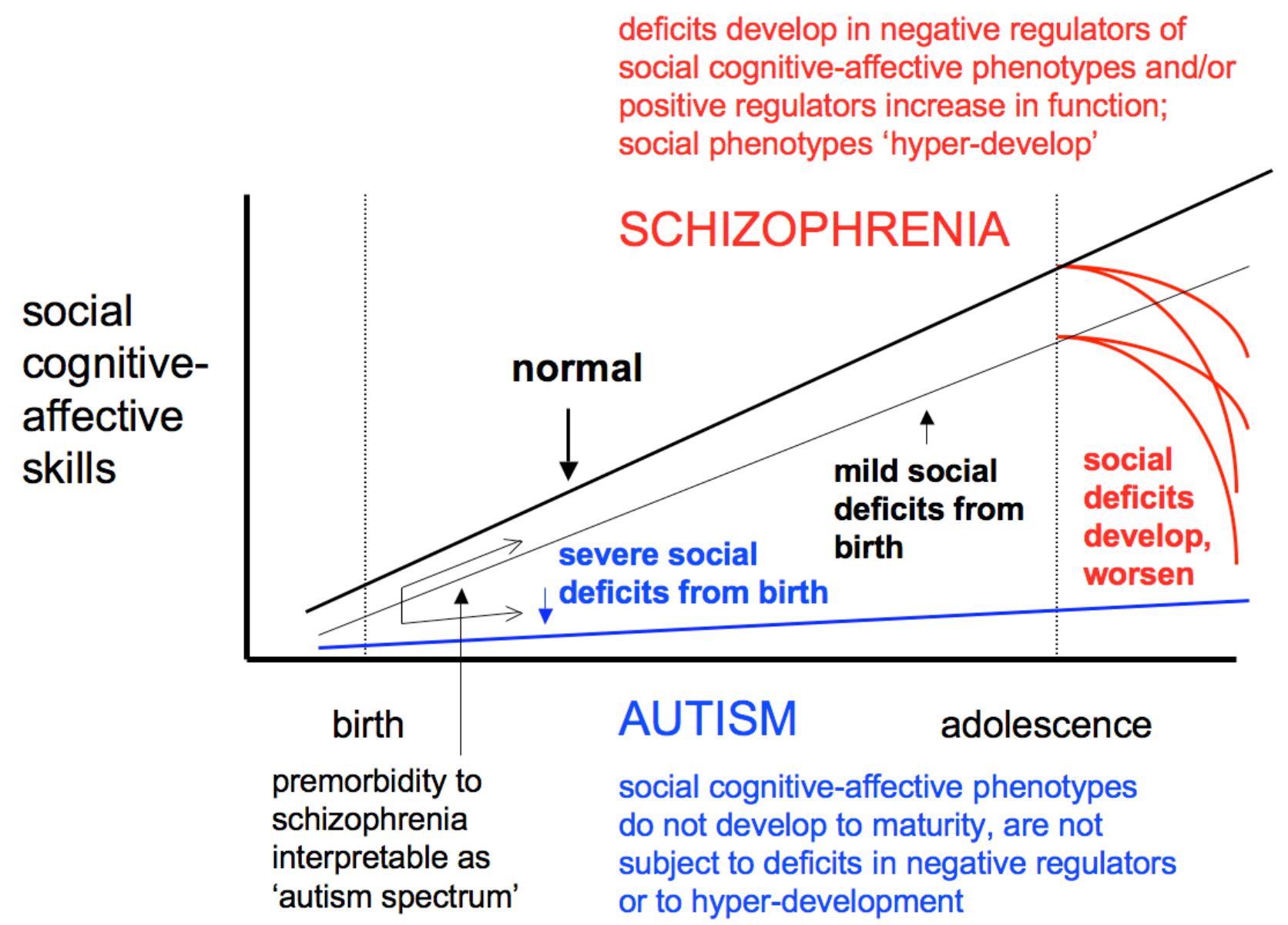


Figure 5. The relationships of autism spectrum and psychotic-affective spectrum phenotypes to social-behavioral-cognitive phenotypes that have become highly developed and elaborated along the human lineage.

\section{AUTISM \\ SPECTRUM \\ PHENOTYPES}

LANGUAGE
SOCIAL
RECIPROCITY
RESTRICTED
INTERESTS,
REPETITIVE
BEHAVIOR

\section{Speech reduced, mechanistic}

Reduced sense of self

Reduced theory of mind

Social emotions underdeveloped

Logic mechanical, inflexible

Reduced goal pursuit

Repetitive thought, tight associations

Mechanistic savant, visual-spatial skills

Social brain hypo-development, due to losses of function in positive regulators of social brain development and/or increased function of negative regulators

\section{HUMAN- ELABORATED PHENOTYPES}

Language
Sense of self
Mentalistic skill
Social emotionality
Causal cognition
Complex goal pursuit
Associational thought
Creativity

Auditory hallucination Megalomania, ideas of reference Paranoia, delusions of persecution Depression, elation Thought disorder

Mania, irritability

Loose associations

Mentalistic verbal skills, alterations

\begin{tabular}{|l} 
SCHIZOPHRENIA, \\
SCHIZOTYPY \\
BIPOLAR \\
DISORDER \\
MAJOR \\
DEPRESSION
\end{tabular}

Social brain 'hyper-development', due to reduced function in negative regulators of social brain functions, and/or increased function of positive regulators 